\title{
Volume Definition Tools for Medical Imaging Applications
}

\author{
Grigoris Karangelis ${ }^{1}$, Stelios Zimeras ${ }^{2}$, Evelyn Firle ${ }^{1}$, \\ Min Wang ${ }^{1}$, Georgios Sakas ${ }^{1}$ \\ ${ }^{1}$ Institut für Graphische Daten Verarbeitung-IGD, Fraunhofer, Darmstadt, Germany \\ \{karangel, efirle, mwang, gsakas\}@igd.fhg.de \\ ${ }^{2}$ MedCom GmbH, Darmstadt, Germany. \\ szimeras@medcom-online.de
}

\begin{abstract}
Central focus of this work, are techniques used to improve time and interaction needed for a user when defining one or more structures. These techniques involve interpolation methods for the manual volume definition and methods for the semi-automatic organ shape extraction.
\end{abstract}

\section{Introduction}

In this work we will present a number of methods that improve the time and interaction one needs to define one or more structures. These techniques involve interpolation methods for the manual volume definition and methods for the semiautomatic organ shape extraction. In the next section the interpolation techniques for the manual volume definition would be described. The methods for the automatic shape extraction of structures are located in section 3, and their comparison in section 4. The work concludes with the discussion section.

\section{Manual Volume Definition}

The manual volume definition is the most common and traditional way to define contours. Usually the user has the possibility to create a contour line using discrete or continuous points. In most systems, the volume definition tools can be applied only on the original, usually axial direction, although images on orthogonal reconstructed planes are provided as well.

Linear Interpolation: Linear interpolation between contours is the first approach used to provide an acceleration tool for the manual contouring. The mechanism of the linear interpolation is applied when between the key contours at least one slice exists. To perform the linear interpolation we create triangles between the contour points of the key contours as described in [1]. For this operation both contour's points must be rotated towards the same rotation direction. The interpolated contour points are created after calculating the intersection of each triangle side with the intermediate slice.

Orthogonal Contour Interpolation: The orthogonal contour interpolation serves to create a volume combining and interpolating orthogonal drawn contours. Principally the algorithm needs at least 2 orthogonal contours to work. The perpendicular plane to these two contours creates intersection points that are the key points to create the new 
interpolated contour. In this approach we use the cubic Spine interpolation. In case the lines are completely equal in size and their centres match or have very small distance then the result of the interpolation will be a circle. In any other case that the two vertical lines are unequal the result will be an ellipse.

\section{Automatic Contouring}

Regarding segmentation techniques, several contour and region approaches have been proposed [3]. The former, are often less robust and more sensitive to noise and variability of data. Hence we will describe and compare in the following section different tools for semi-automatic contouring. For the visual comparison of the semiautomatic techniques, the EXOMIO ${ }^{1}$ virtual simulation package was used.

Boundary tracking technique: Boundary tracking techniques is a segmentation method, that given one point along a region's boundary follows the boundary around the region until it returns to the original point. The advantage is that no assumptions need be made a priori about the boundary shape. The algorithm starts with an initial point. Different starting directions are defined until a sharp edge was found. The tracking procedure stops only when passing through the starting point in the original contour direction.

Active Contour Model: Active Contour Models (ACMs) are adaptive contour representations, also known as snakes or deformable models. They are able to recover and represent physical contours of an image, and hence can be used as a model to determine object boundaries in static images as well as for tracking in image sequences. Grosskopf et al. [4] uses the Euler Time Integration to solve the optimisation problem. After initialisation by a user sketch, the contour is deformed to fit the actual object by simulating physical properties of an elastic material or fluid. This method is very reliable to overcome local minima and very fast due to its deterministic character.

\section{Comparison of the Semi-automatic Methods}

Materials and Measures: The two proposed contouring methods have been applied for a CT tomographic sequence of 8 bit gray scale images of a size $512 \times 512$ in which change the shape, and the orientation of the objects. In this work, segmentation results would be presented for three different regional objects: (a) concave (lungs), (b) convex (lungs), (c) large (skin), (d) small (marrow of the spinal canal). To assess the effectiveness of the methods and its sensitivity, the following object measures are considered: (1) area of a contour, and (2) periphery of a contour.

Segmentation results: The visual comparison of the segmented areas between the boundary tracking (green line-2) and the active contour (red line-1) methods are given in Figure 1 for all the different regions. It is clear that for large segmented areas (Figure 1c) and convex regions (Figure 1b) both methods gives satisfactory results. The contour edges are defined approximate correctly with few false alarm for the active contour technique due to the edge over-smoothness of the process. Different results was found in cases where the areas are concave (Figure 1a) or small and open

\footnotetext{
${ }^{1} \mathrm{http}: / /$ www.medcom-online.de/
} 
(Figure 1d). For the first case, the segmentation using the active contour method is unsatisfactory compared with the boundary tracking method. The regions, where sharp edges were appeared, were over-smoothed or disappeared. For the second case, segmentation results suggest that when the areas are small and open the boundary tracking technique was unable to find the appropriate region with false identification of the areas. Alternatively, the active contour technique gives satisfactory results, where the shape of the region was defined correctly.
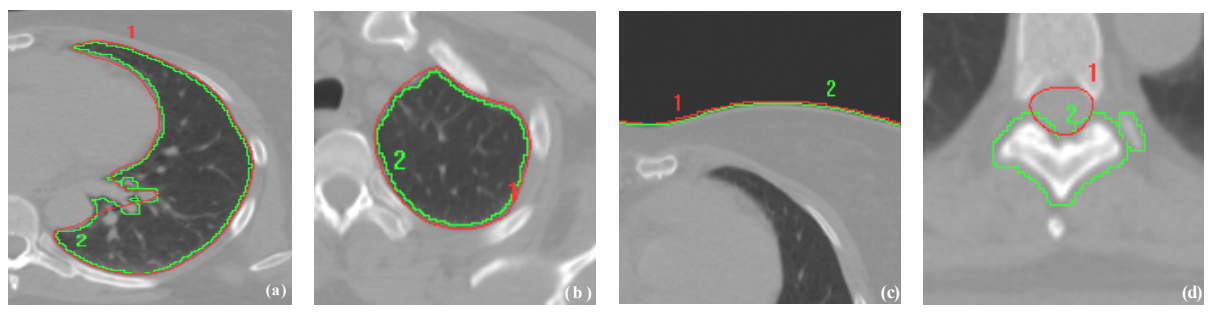

Fig. 1. Visual comparison for different regions; Red line (1): Active contour method, Green line (2): Boundary tracking method. (a) concave (lungs), (b) convex (lungs), (c) large (skin), (d) small (spinal canal marrow).

\section{Discussion}

In this work different volume definition techniques has been presented. In the first part we present interpolation techniques aim to accelerate the manual contouring process. That includes the well-known linear interpolation technique implemented for simple bisection organ shapes. In addition we introduce a new interpolation method, the orthogonal interpolation, which enables the user to define a volume. At the second part, two contour tracking techniques has been analysed; a boundary tracking and an active contour method. The algorithms have been tested on different regions. For large and convex regions both methods work satisfactory. For concave regions, boundary tracking technique works better compared to the active contour technique. Areas with discontinuities and sharp edges are defined successfully for the boundary tracking method where for the active contour a few false edges are presented. Finally, for small and open regions, active contour method was able to segment the areas, where the boundary tracking method fails.

\section{References}

1. Ekoule A, Peyrin F.: A Traingulation Algorithm from Arbitrary Shaped Multiple Planar Contours, ACM Trans. On Graphics, Vol. 10, 1991.

2. Haralick R. M. and Shapiro L. G.: Image segmentation techniques, Comput. Vis. Graph. Im. Proc., 29:100-132, 1985.

3. Großkopf S, Park SY, and Kim MH: An improved Active Contour Model for Segmentation of Medical Images, Proc. $3^{\text {rd }}$ Korea-Germany Joint Conference on Advanced Medical Image Processing, Seoul, Korea, 1998. 